\title{
Asiatische Wachstumsmärkte und deutsche Exporte - unter besonderer Berücksichtigung des Landes Brandenburg
}

\author{
Prof. Dr. Bernd Wonneberger, Dipl.-Psych. Hans Könecke
}

\section{Problemstellung}

Seit 1990 haben in Mittel- und Osteuropa gravierende gesellschaftliche und wirtschaftliche Veränderungen stattgefunden, die in den neuen Bundesländern u. a. einen erheblichen Rüickgang der Arbeitsplätze im produzierenden bzw. verarbeitenden Gewerbe verursacht haben. Viele dieser Unternehmen wurden aufgelöst, die verbleibenden Unternehmen haben ihre Belegschaften zumeist stark reduziert. Kenntnisreich und detailliert wurde dieser Prozeß beispielsweise geschildert in einem Referat des Geschäftsführers der SMB Wildau GmbH anläßlich des Besuches einer Delegation des chinesischen Verbandes für Unternehmensführung der Kohleindustrie.

Die Zahl der Beschäftigten im produzierenden und verarbeitenden Gewerbe der ehemaligen DDR betrug im Jahre 1988 rund 2,7 Millionen; in den neuen Bundesländern betrug diese Zahl im Jahre 1996 noch knapp 800.000 Beschäftigten. Vor allem wegen der geringen Zahl an Unternehmen im produzierenden und verarbeitenden Gewerbe ist der Anteil der neuen Bundesländer am gesamten deutschen Außenhandel, im Vergleich zu anderen Bundesländern wie beispielsweise Baden-Württemberg oder Nordrhein-Westfalen, nach wie vor gering (s. unten, Abschnitt 2). An der Entwicklung des gesamten Welthandels, welche in der Abbildung 1 dargestellt wird, haben die beiden Bundesländer Brandenburg und Berlin, wie Deutschland insgesamt, in den vergangenen Jahren nur unterdurchschnittlich partizipiert.

„Nach Angaben des Instituts der deutschen Wirtschaft stiegen zwar die deutschen Exporte von 1989 bis 1996 um 14 Prozent. Allerdings wuchsen die Absatzmärkte im gleichen Zeitraum um reichlich 46 Prozent ... Laut DIHT ist der Rückgang des deutschen Marktanteils unter anderem auch dadurch begründet, daß die deutsche Exportwirtschaft auf wachstumsstarken Märkten - etwa in Asien - relativ schwach vertreten ist" (Berliner Zei-

Deutsche Exporteure schöpfen wachsende Märkte nicht aus (in \%, $1989=100$ )

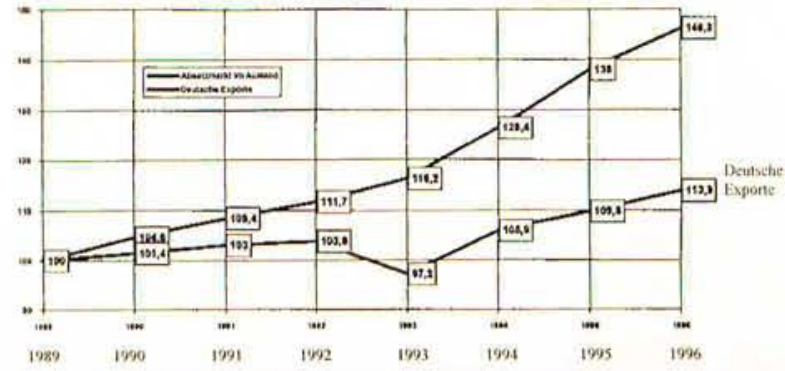

Abb. 1: Entwicklung des gesamten Welthandels und der deutschen Ausfuhren von 1989 bis 1996 (Berliner Zeitung vom 18.8.1997 nach Angaben des StBA) tung, 18.8.1997). Dagegen hat sich der Handel in der Asien-Pazifik-Region oder in Lateinamerika in den vergangenen Jahren wesentlich stärker als in Europa entwickelt.

Die geschilderten Defizite bei den deutschen, vor allem den brandenburgischen Exporten waren der Ausgangspunkt des Forschungsprojektes „Markterschließung in Ost- und Südostasien für kleine und mittlere Unternehmen (KMU) in betrieblicher Kooperation und Erprobung praxisnaher Methoden der Fachhochschulausbildung", das die Autoren dieses Beitrages zusammen mit anderen Kollegen und den Studierenden an der Technischen Fachhochschule Wildau vom 1.10 .1996 bis zum 30.11.1997 durchführten.

Erste Ergebnisse dieses Projektes wurden vorgestellt unter dem Titel „Asien - Wachstumsmärkte für brandenburgische Unternehmen?" (KÖNECKE et al. 1997). Mehrere Fragezeichen hinter diesen Titel zu stellen, mag manchem Leser angemessen erscheinen nach der Entwicklung des Jahres 1997. Viele Volkswirtschaften Ostund Südostasiens hatten und haben weiterhin enorme Probleme mit großen Defiziten in den Staatshaushalten, deren Anteile am Bruttoinlandsprodukt der asiatischen Länder oft wesentlich über der „Euro-Norm“ von drei Prozent liegen.

Weiterhin war das Wirtschaftsjahr 1997 in Indonesien, Japan, Korea, Malaysia, Thailand und anderen Staaten geprägt von den Konkursen diverser Banken und großen Kursverlusten an den Aktienmärkten. Ausgenommen von dieser Entwicklung blieb zunächst die Volksrepublik China, die seit 15 Jahren Wachstumsraten ihres Bruttoinlandsproduktes von 8 bis 13 Prozent verzeichnet und eine auf 7 Prozent zurüickgeführte jährliche Inflationsrate ausweist. Am Ende des Jahres 1997 verfügte die VR China über Devisenreserven von rund 115 Milliarden US Dollar.

Die wirtschaftlichen Probleme Japans werden nach unserer Auffassung gleichfalls nur vorübergehender Natur sein, wenn erst einmal die Ende 1997 konzipierten Programme zur Stärkung der japanischen Binnenkonjunktur und zur Verringerung des Staatsdefizites größere Wirkungen zeigen (vgl. ausfuihrlich dazu der Beitrag von BRUNSCH in diesem Heft). In ähnlicher Weise werden auch andere asiatische Länder in den nächsten Jahren alle Anstrengungen unternehmen, welche nach einer kurzen Phase der Konsolidierung die Basis für weiteres Wachstum im Asien-Pazifik-Raum bilden wird.

Angesichts der kurz beschriebenen Entwicklung des Jahres 1997 wollen die Autoren im folgenden diskutieren, ob ein starkes Engagement in Asien für brandenburgische Unternehmen nach wie vor sinnvoll sein könnte. 


\section{Betrachtungen zur Außenhandelsstatistik Brandenburgs und Deutschlands}

Nach der Außenhandelsstatistik des Landesantes für Datenverarbeitung und Statistik betrugen die Ausfuhren Brandenburgs im Jahr 1991 knapp 2,4 Mrd. DM, im Jahre 1996 betrugen sie zuletzt 4,2 Mrd. DM (vgl. Tabelle 1).

\begin{tabular}{|llcc|}
\hline Jahr & $\begin{array}{l}\text { Summe } \\
\text { in TDM }\end{array}$ & $\begin{array}{c}\text { Gewerbliche } \\
\text { Wirtschaft }\end{array}$ & $\begin{array}{c}\text { davon: } \\
\text { Eimährungs- } \\
\text { wirtschaft }\end{array}$ \\
\hline 1991 & 2.376 .572 & 2.188 .800 & 187.772 \\
1992 & 2.603 .991 & 2.261 .361 & 342.630 \\
1993 & 2.502 .050 & 2.186 .280 & 315.770 \\
1994 & 2.820 .082 & 2.543 .578 & 276.504 \\
1995 & 3.541 .731 & 3.232 .547 & 309.184 \\
1996 & 4.231 .949 & 3.886 .834 & 345.115 \\
\hline
\end{tabular}

Tab. I: Ausfulren des l.andes Brandenburg 1991-1996 in TDM (L.ADVSTAT 1997)

Dieser auch unter Einrechnung von Preissteigerungen noch erkennbare Anstieg der Brandenburger Ausfuhren basiert auf einem vergleichsweise sehr geringen Niveau. Im Jahre 1996 betrugen die gesamten Ausfuhren der Bundesrepublik Deutschland knapp 772 Mrd DM. Somit hatte Brandenburg zuletzt einen Anteil von gerade einmal 0,55 Prozent an den gesamten deutschen Ausfuhren, welcher in Tabelle 2 ausgewiesen wird (StBA 1997a, S. 36).

\begin{tabular}{|lccccccc|}
\hline Jahr & $\begin{array}{c}\text { Branden- } \\
\text { burg }\end{array}$ & Berlin & \& & $\begin{array}{c}\text { Gesamt- } \\
\text { region }\end{array}$ & $\begin{array}{c}\text { BRD } \\
\text { gesamt }\end{array}$ \\
\hline 1991 & 2.377 & 0.36 & 14.072 & 2.11 & 16.449 & 2.47 & 665.813 \\
1992 & 2.604 & 0.39 & 12.034 & 1.79 & 14.638 & 2.18 & 671.203 \\
1993 & 2.502 & 0.40 & 11.390 & 1.81 & 13.892 & 2.21 & 628.387 \\
1994 & 2.820 & 0.41 & 12.124 & 1.76 & 14.944 & 2.16 & 690.573 \\
1995 & 3.542 & 0.49 & 11.939 & 1.64 & 15.481 & 2.13 & 727.732 \\
1996 & 4.232 & 0.55 & 12.483 & 1.62 & 16.715 & 2.17 & 771.913 \\
\hline
\end{tabular}

Tab. 2: Anteile von Brandenburg und Berlin an den gesamten Ausfuhren der Bundesrepublik Deutschland (StBA 1997a, S. 36)

Die Verlagerung von Produktionsstätten, zumeist von Berlin nach Brandenburg, findet an dieser Stelle ihren Ausdruck auch in der Statistik der Ausfuhren. Letztlich stagniert das Volumen der Ausfuhren aus der Gesamtregion auf einem niedrigen Niveau. Dies wiederum hat für die Wirtschaftsentwicklung und den Arbeitsmarkt in der Region Brandenburg und Berlin fatale Konsequenzen. In den Jahren 1991 bis 1996 konnte die Bundesrepublik Deutschland ihre Ausfuhren um 106.000 Mio. DM steigern. Der größte Teil der Konjunkturimpulse kam in diesen Jahren vom deutschen Export, wobei aber Brandenburg und insbesondere Berlin von dieser Entwicklung kaum profitieren konnten. Andere Bundesländer hatten weit höhere Anteile am deutschen Export und seinen Zuwachsraten, wie wir für das Jahr 1996 in Tabelle 3 darstellen.

\begin{tabular}{|c|c|c|c|c|c|}
\hline 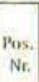 & Bundestand & $\begin{array}{l}\text { Ausfultr in } \\
\text { Mis DM }\end{array}$ & $\therefore$ & $\begin{array}{l}\text { Linwoluer } \\
\text { in Tst, per } \\
31.12 .1995\end{array}$ & $\begin{array}{c}\text { Erwertbstatige } \\
\text { in Tsd. per } \\
30.04 .19 \% 6\end{array}$ \\
\hline 1. & Nordrhein-Westfalen & 165.455 & 21,43 & 17.893 & 7.341 \\
\hline 2. & Baden-Württemberg & 131.386 & 17,02 & 10.319 & 4.764 \\
\hline 3. & Bayern & 118.316 & 15,33 & 11.993 & 5.744 \\
\hline 4. & Niedersachsen & 55.486 & 7.19 & 7.780 & 3.356 \\
\hline 5. & Hessen & 48.621 & 6,30 & 6.010 & 2.689 \\
\hline 6. & Rheinland-Pfalz. & 37.831 & 4,90 & 3.978 & 1.714 \\
\hline 7. & Hamburg & 19.436 & 2,52 & 1.708 & 769 \\
\hline 8. & Bremen & 13.597 & 1,76 & 680 & 280 \\
\hline 9. & Berlin & 12.483 & 1.62 & 3.471 & 1.573 \\
\hline 10. & Schleswig-Holstein & 11.493 & 1.49 & 2.725 & 1.250 \\
\hline 11. & Saarland & 11.308 & 1.46 & 1.084 & 413 \\
\hline 12. & Sachsen & 7.186 & 0.93 & 4.567 & 1.962 \\
\hline 13. & Thüringen & 4.452 & 0,58 & 2.504 & 1.082 \\
\hline 14. & Brandenburg & 4.232 & 0.55 & 2.542 & 1.100 \\
\hline 15. & Sachsen-Anhalt & 4.228 & 0.55 & 2.739 & 1.142 \\
\hline 16. & Mecklenburg-Vorpommern & 2.015 & 0.26 & 1.823 & 803 \\
\hline & Summe & 647.525 & 83,89 & 81.817 & 35.982 \\
\hline
\end{tabular}

Tab. 3: Anteile der 16 Bundesländer an den Ausfuhren 1996 der BRD in Mio. DM (StBA 1997a, S. 32-37)

Erläuterung zu Tabelle 3: Die Werte in Prozent beziehen sich auf die Gesamtsumme der Ausfuhren von 771.993 Mio DM. Die Differenz zu 100 Prozent entsteht durch jene Ausfuhren. die in der Statistik wegen fehlender Angaben in der Zollerklärung keinem der 16 Bundesländer eindeutig zugeordnet werden konnten.

Das Verhältnis der Ausfuhren zur Zahl der Einwohner oder der Erwerbstätigen ist in allen neuen Bundesländern deutlich geringer als in den alten Bundesländern. Selbst das Saarland erzielte mit 413.000 Erwerbstätigen Ausfuhren im Wert von 11.308 Mio. DM; das ist weitaus mehr als jene 4.232 Mio. DM, welche Brandenburg mit 1.100.000 Erwerbstätigen erzielen konnte. Diese Vergleiche zeigen einmal mehr, daß nicht nur in Brandenburg, sondern in allen neuen Bundesländern, nach unserer Ansicht folgende Aktivitäten der Wirtschaftsförderung notwendig sind:

1. Weitaus mehr Unternehmen des verarbeitenden und produzierenden Gewerbes, die Produkte für den Weltmarkt herstellen, müssen angesiedelt oder durch Existenzgründung geschaffen werden.

2. Die internationale Wettbewerbsfähigkeit der bereits existierenden Unternehmen verschiedenster Wirtschaftszweige sollte entscheidend verbessert werden.

3. International wettbewerbsfähige Unternehmen sollten dazu angeregt und unterstiitzt werden, weltweit Wachstumsmärkte konsequent zu erschließen. Auch hierbei hat Brandenburg einen Rückstand gegenüber den Unternehmen anderer Bundesländer. Etwa 35 Prozent aller deutschen Ausfuhren gingen im Jahr 1995 in Länder außerhalb Europas (BMWI 1997, S. 89), während deren Anteil in Brandenburg nur 20 Prozent betrug (vgl. unten, Tabelle 5). 
Im folgenden geben wir einen Überblick über die Zielländer und -kontinente der Ausfuhren aus Brandenburg in den Tabellen 4 und 5.

\begin{tabular}{|c|c|c|c|c|c|c|}
\hline \multirow{3}{*}{$\begin{array}{l}\text { Stume in TDM } \\
\text { Zielland } \\
\text { Niederlande }\end{array}$} & \multirow{2}{*}{\multicolumn{2}{|c|}{$\begin{array}{l}3.541 .731 \quad \text { Anteil } \\
\\
1995 \text { in : }\end{array}$}} & \multirow{2}{*}{$\begin{array}{l}\text { Veräinderung } \\
\text { Zu } 1994 \text { in \$ }\end{array}$} & \multirow{2}{*}{\multicolumn{2}{|c|}{$\begin{array}{r}4.231 .949 \text { Anteil } \\
1996 \mathrm{int}:\end{array}$}} & \multirow{2}{*}{$\begin{array}{l}\text { Verainderung } \\
\text { za } 1995 \text { in: }\end{array}$} \\
\hline & & & & & & \\
\hline & 425.964 & 12,03 & 34,2 & 432.455 & 10.22 & 1,5 \\
\hline Polen & 259.957 & 7,34 & 38.7 & 347.629 & 8,21 & 33,7 \\
\hline Frankreich & 258.792 & 7,31 & 22,2 & 284.127 & 6,71 & 9,8 \\
\hline USA & 255.018 & 7.20 & 48,4 & 530.500 & 12,54 & 108,0 \\
\hline $\begin{array}{l}\text { Rußland } \\
\text { ferner u.a. } \\
\text { Tschechische }\end{array}$ & 251.277 & 7,09 & 6,2 & 214.831 & 5,08 & $-14,5$ \\
\hline $\begin{array}{l}\text { Tschechische } \\
\text { Republik }\end{array}$ & 90.097 & 2.54 & 63,0 & 105.504 & 2,49 & 17.1 \\
\hline Usbekistan & 54.778 & 1.55 & 689,3 & 44.438 & 1,05 & -18.9 \\
\hline Thailand & 40.775 & 1,15 & 489,8 & 11.431 & 0,27 & $-72,0$ \\
\hline Israel & 34.189 & 0.97 & $-23,4$ & 26.895 & 0.64 & $-21,3$ \\
\hline Saudi-Arabien & 28.240 & 0.80 & 691,7 & 13.993 & 0,33 & -50.4 \\
\hline China & 25.520 & 0,72 & $-44,9$ & 45.244 & 1.07 & 77,3 \\
\hline Hongkong & 20.085 & 0,57 & $-58,2$ & 33.184 & 0,78 & 65,2 \\
\hline Indonesien & 19.873 & 0.56 & 209,0 & 8.408 & 0,20 & $-57,7$ \\
\hline Japan & 19.029 & 0,54 & 84,1 & 16.318 & 0,39 & -14.2 \\
\hline Indien & 17.354 & 0.49 & $-3,8$ & 32.411 & 0,77 & 86,8 \\
\hline Malaysia & 16.570 & 0.47 & 14,0 & 14.629 & 0.35 & $-11,7$ \\
\hline Taiwan & 14.636 & 0.41 & 80,5 & 12.397 & 0.29 & $-15,3$ \\
\hline $\begin{array}{l}\text { Vereinigte Ara- } \\
\text { bische Emirate }\end{array}$ & 14.613 & 0.41 & $-27,7$ & 5.853 & 0,14 & $-59,9$ \\
\hline Singapur & 11.670 & 0,33 & 71,0 & 18.035 & 0,43 & 54,5 \\
\hline
\end{tabular}

Tabelle 4: Ausfuluren Brandenburgs nach ausgewählten Bestimmungsländern in den Jahren 1995 und 1996 (nach Angaben LADVSTAT 1997)

Bei vielen der in Tabelle 4 genannten Bestimmungsländern erkennen wir, daß die brandenburgischen Ausfuhren dorthin von Jahr zu Jahr starken Schwankungen unterliegen. Dies liegt hauptsächlich am geringen Volumen der Ausfuhren in Bestimmungsländer außerhalb Europas. So stiegen beispielsweise im Jahre 1995 die Ausfuhren nach Usbekistan, Thailand oder Saudi-Arabien prozentual stark an, im Jahre 1996 gingen sie wiederum stark zurück. Einige größere Aufträge zum Beispiel in der Kraftwerks- oder Verkehrstechnik können bei dem jeweils geringen Volumen der Ausfuhren bereits erhebliche (negative wie positive) Veränderungen bewirken. Wir befassen uns deshalb im weiteren beim Kommentar zur Tabelle 4 mit jenen fünf Ländern, die im Jahre 1996 die größten Anteile hatten am Volumen der brandenburgischen Ausfuhren.

An erster Stelle waren dies die USA mit einem Anteil von 12,54 Prozent - eine Entwicklung, die bis dato nur selten von der regionalen Presse wahrgenommen wurde. Danach folgten die Niederlande (Anteil 10,22\%), Polen $(8,8 \%)$ und Frankreich $(6,71 \%)$, wobei insbesondere das Volumen der Ausfuhren nach Polen und Frankreich gegenüber 1995 zugenommen hatte. Dagegen ging das Volumen der Ausfuhren nach Rußland im Jahre 1996 um 36,5 Millionen DM zurück. Nach den vorläufigen Zahlen der Außenhandelsstatistik 1997, welche die Autoren hier noch nicht veröffentlichen wollten, setzten sich die skizzierten Entwicklungen im Jahre 1997 fort.

Dies nehmen die Autoren zum Anlaß, kritisch zu hinterfragen, ob die nach wie vor auch von der Presse transportierte Ansicht, „Rußland sei ein naturgemäßer und wichtiger Partner für die brandenburgische Wirtschaft", zutrifft. Dabei wird nach unserer Auffassung leichtfer- tig übersehen, daß die Bonität Rußlands nahezu erschöpft ist. Nach den Umschuldungen der vergangenen Jahre hat Rußland gegenüber seinen internationalen Gläubigern rund $80 \mathrm{Mrd}$. DM Kreditverbindlichkeiten. Die Grenze der russischen Kreditfähigkeit dürfte mit weiteren $15 \mathrm{Mrd}$. DM erreicht werden. In der Konsequenz heißt dies für brandenburgische Unternehmen, daß für Geschäfte mit russischen Partnern in den nächsten Jahren kaum Devisen oder Devisenkredite zur Verfuigung stehen. Eine Finanzierung der Geschäfte ist dann nur noch in Form von Bartergeschäften möglich, sofern eine Barzahlung nicht erreicht werden kann.

\begin{tabular}{|c|c|c|c|c|c|c|}
\hline Kontinent & $\begin{array}{r}1995 \\
\text { in TDM }\end{array}$ & $\begin{array}{c}\text { Anteil } \\
\text { in: }\end{array}$ & $\begin{array}{l}\text { Veränderung } \\
\text { zu } 1994 \text { in th }\end{array}$ & $\begin{array}{r}1996 \\
\text { in } \mathrm{TDM}\end{array}$ & $\begin{array}{c}\text { Anteil } \\
\text { in : }\end{array}$ & $\begin{array}{l}\text { Veränderung } \\
\text { xu } 1995 \text { in : }\end{array}$ \\
\hline Europa & 2.843 .568 & 80,3 & 27.9 & 3.227 .555 & 76,3 & 13,5 \\
\hline Amerika & 332.129 & 9.4 & 33,9 & 612.371 & 14.5 & 84,4 \\
\hline Asien & 305.526 & 8.6 & 5,3 & 301.066 & 7.1 & -1.5 \\
\hline Afrika & 47.968 & 1,4 & $-6,9$ & 71.824 & 1,7 & 49.7 \\
\hline $\begin{array}{l}\text { Australien u. } \\
\text { Ozeanien }\end{array}$ & 11.398 & 0,3 & 83,1 & 17.258 & 0,4 & 51.4 \\
\hline Verschiedene & 1.142 & 0,0 & 71,2 & 1.875 & 0,0 & 64,2 \\
\hline Summe & 3.541 .731 & 100,0 & & 4.231 .949 & 100,0 & \\
\hline
\end{tabular}

Tab. 5: Ausfuhren Brandenburgs nach den fünf Erdteilen in den Jahren 1995 und 1996, (nach Angaben LADVSTAT 1997)

Wir können über den dargestellten Zeitraum (Tabellen 4 und 5) für die brandenburgischen Ausfuhren folgende Entwicklungen feststellen:

1. Das Volumen der Ausfuhren in die Länder der Europäischen Union wuchs kontinuierlich. Ferner sind die USA - zumindest im Jahre 1996 - der größte Abnehmer geworden für Ausfuhren aus Brandenburg.

2. In Mitteleuropa entwickelten sich die Ausfuhren Brandenburgs nach Polen und in die Tschechische Republik positiv. Bei anderen Ländern Mittel- und Osteuropas war die Entwicklung negativ, in Tabelle 4 wurde hier nur Rußland beispielhaft dargestellt und interpretiert.

3. Die Ausfuhren aus Brandenburg nach Asien insgesamt haben nach wie vor nur ein geringes Volumen. Positive und negative Entwicklungen der einzelnen asiatischen Ländermärkte glichen sich aus brandenburgischer Sicht im Ergebnis der Jahre 1995 und 1996 nahezu aus. Ob sich die positive Entwicklung der Ausfuhren des Jahres 1996 in die VR China und Hongkong, Indien und Singapur auch in den nächsten Jahren fortsetzen läßt, ist abhängig von den konkreten Aktivitäten der brandenburgischen Unternehmen auf diesen asiatischen Märkten. Die Attraktivität dieser Märkte wird, nach Auffassung der Autoren, mittelfristig weiter wachsen, sobald die Konsolidierungsprogramme der dortigen Regierungen Erfolg hatten.

Gemäß der Zielstellung unseres Forschungsprojektes wollten die Autoren für kleine und mittlere Unternehmen (KMU) des Landes Brandenburg Hilfen zur Erschließung der Märkte in Ost- und Südostasien erarbeiten. Dadurch ließen sich in den nächsten Jahren die im letzten Absatz diskutierten Ausfuhren nach Asien stabilisieren, vielleicht sogar noch steigern. Nach den Ergebnissen unseres Forschungsprojektes (welche auch in ande- 
ren Aufsätzen dieses Heftes der Wissenschaftlichen Beiträge dargestellt werden) können wir sagen, daß im Jahre 1997 nur wenige brandenburgische Unternehmen über eine klar beschriebene Strategie zur Erschließung und Bearbeitung von Märkten in Ost- oder Südostasien verfügten. An jede Art von Beratung und Förderung von Unternehmen zur Erschließung asiatischer Märkte sind deshalb a priori folgende zwei Anforderungen zu stellen:

1. Identifizierung attraktiver Ländermärkte in Asien,

2. Formulierung einer internationalen Marktingstrategie adäquat zum Unternehmen und zum Zielmarkt.

Allgemeine Ausführungen zur Formulierung einer internationalen Marketingstrategie halten die Autoren im Rahmen dieses Beitrages nicht für sinnvoll, angesichts der Vielzahl von Faktoren des Unternehmens und des Marktes, welche die Wahl der Strategie letztlich bestimmen (vgl. dazu HÜNERBERG 1994, MEISSNER 1987, MEYER 1987, MUNKELT 1996, NIESCHLAG/DICHTL/ HÖRSCHGEN 1991, PORTER 1995, STAHR 1993).

Wir befassen uns im folgenden Abschnitt abschließend mit der Frage, ob und wenn ja, welche Ländermärkte in Ost- und Südostasien in den nächsten Jahren für brandenburgische Unternehmen attraktiv sein könnten.

\section{Perspektiven für Brandenburger Unter- nehmen auf Märkten in Ost und Südost- asien}

\subsection{Bevölkerungsentwicklung und wirtschaftliche Trends in den Staaten Ost- und Südostasiens}

Die im ersten Abschnitt nur kurz für den asiatisch-pazifischen Raum skizzierten wirtschaftlichen Probleme im Herbst 1997 sind in den deutschen Medien ausführlich dargestellt worden. Die Autoren wollen an dieser Stelle erörtern, welche Entwicklungen bis zum Jahr $2000 \mathrm{im}$ asiatisch-pazifischen Raum möglich sind. In ihrem Bericht über die internationale Fachausstellung BauconAsia vom 7. bis 10. Oktober 1997 in Singapur kam zum Beispiel die BfAi zu folgenden Aussagen: „Die „Großbaustelle Asien" mit einem potentiellen Auftragsvolumen von $900 \mathrm{Mrd}$. US-Dollar bleibt den Lieferanten von Bautechnik jedoch erhalten. Anders als bisher erstreckt sich dieses Ordervolumen noch über das Jahr 2000 hinaus, wovon annähernd die Hälfte auf die zur suidostasiatischen Wirtschaftsgruppierung ASEAN ... zählenden Länder Brunei, Indonesien, Laos, Malaysia, Myanmar, Philippinen, Singapur, Thailand und Vietnam entfällt" (BfAi 1997, S. 1).

Selbst unter erschwerten finanzpolitischen Bedingungen werden diese Länder nach Auffassung der Autoren in den nächsten Jahren große Investitionen tätigen, um die Grundbedürfnisse einer stetig wachsenden Bevölkerung decken zu können (vgl. Tabelle 6). Weitere Infrastrukturmaßnahmen sind notwendig, um durch die Modernisierung der Energieversorgung, des Verkehrswesens, der Telekommunikation und letztlich auch der Produktion in den Unternehmen die Basis für ein weiteres wirtschaftliches Wachstum zu schaffen.

\begin{tabular}{|c|c|c|c|c|}
\hline Stat & $\begin{array}{l}\text { Hijche } \\
\text { in qkm }\end{array}$ & $\begin{array}{l}\text { Bevölkerumg } \\
1996 \\
\text { in Tsd. }\end{array}$ & $\begin{array}{l}\text { Bevölkerumg } \\
1996 \\
\text { je qkm }\end{array}$ & $\begin{array}{c}\text { Wachstumsrate } \\
\text { der Bevolkerung } \\
1990-1995\end{array}$ \\
\hline \multicolumn{5}{|l|}{ ASE:AN-Staoten } \\
\hline Indonesien & 1.904 .569 & 200.596 & 105 & 1.55 \\
\hline Malaysia & 329.758 & 20.581 & 62 & 2,37 \\
\hline Philippinen & 300.000 & 68.976 & 230 & 2.12 \\
\hline Singapur & 618 & 2.874 & 4.650 & 1.03 \\
\hline Thailand & 513.115 & 59.414 & 116 & 1.12 \\
\hline Vietnam & 331.689 & 76.161 & 230 & 2,23 \\
\hline \multicolumn{5}{|l|}{ Andere asiatische Staaten } \\
\hline China & 9.560 .961 & 1.212 .985 & 127 & 1,11 \\
\hline Indien & 3.287 .590 & 952.969 & 290 & 1,91 \\
\hline Japan & 377.801 & 125.382 & 332 & 0,25 \\
\hline Republik Korea & 99.274 & 45.429 & 458 & 0,97 \\
\hline Taiwan & 36.000 & 21.353 & 593 & k.A. \\
\hline \multicolumn{5}{|l|}{ zum Vergleich } \\
\hline BR Deutschland & 357.022 & 81.817 & 229 & 0.55 \\
\hline
\end{tabular}

Tab. 6: Bevölkerungsdaten ausgewählter asiatischer Staaten im Vergleich zur Bundesrepublik Deutschland (StBA 1997b, S. 194-207)

Erläuterung zu Tabelle 6: Die Angaben zur Bevölkerung im Jahre 1996 und den Wachstumsraten der Jahre 1990 - 1995 basieren auf Hochrechnungen, nicht auf Volkszählungen.

In Tabelle 6 kann man bei den Wachstumsraten der Jahre 1990 bis 1995 erkennen, daß die Bevölkerung etlicher asiatischer Staaten stark wächst. Am Beispiel Indonesiens sei dieses Wachstum noch einmal verdeutlicht: Eine jährliche Wachstumsrate von 1,55 Prozent bedeutet eine Vergrößerung der Bevölkerung Indonesiens um zirka 3 Millionen Menschen pro Jahr. Übertragen auf die heimischen Verhältnisse würde diese Wachstumsrate bedeuten, daß sich die Bevölkerung Brandenburgs innerhalb eines Jahres verdoppelt.

Die große und rasch wachsende Bevölkerung im asiatisch-pazifischen Raum ist jedoch in etlichen der in Tabelle 6 genannten Staaten kein großes Kaufkraftpotential, da die Pro-Kopf-Einkommen noch sehr gering sind. Eine positive Entwicklung des privaten Konsums erwarten die Autoren nach den wirtschaftlichen Turbulenzen des Jahres 1997 erst nach der durch den Internationalen Währungsfond (IWF) veranlaßten Konsolidierungsphase.

Zur Begründung ihrer Auffassung greifen die Autoren auf eine Analogie der Wirtschaftsgeschichte zurück. In Lateinamerika wurden durch die Regierungen, Unternehmen und Privathaushalte seit Mitte der 60er Jahre gleichfalls Milliardenkredite aufgenommen (vgl. dazu NEEBES 1991). In den Jahren 1984 und 1985 waren einige Länder Lateinamerikas, beispielsweise Mexiko oder Venezuela, so gut wie zahlungsunfähig. Diese Länder mußten ihre Wirtschaftspolitik den Auflagen des IWF und der Gläubigerbanken anpassen, weshalb einige Sozialprogramme drastisch gekürzt wurden, während die Wirtschaft der lateinamerikanischen Staaten stimuliert wurde, ihr Angebot für den Weltmarkt zu erhöhen, um letztlich mit den erwirtschafteten Devisen Kredite bei den internationalen Gläubigern tilgen zu können.

Ende Juni 1996 hatten, nach Angaben der Zahlungsbilanzstatistik der Deutschen Bundesbank, die deutschen Banken Nettoforderungen von 69,2 Milliarden DM an die asiatischen Länder (vgl. Berliner Zeitung vom 28.11.1997, S. 30). Den Schuldendienst für diese Kredite werden die asiatischen Staaten nur erbringen kön- 
nen, wenn sie auf der einen Seite den Import von ausländischen (Konsum-)Gütern reglementieren, auf der anderen Seite ihre Exporterlöse auf dem Weltmarkt steigern. Eine Steigerung der Exporterlöse erfordert wiederum vielfach eine deutliche Erhöhung der Produktivität in den Unternehmen, ebenso wie eine Veränderung des Sortimentes hin zu weltmarktfähigen Produkten.

Unsere skizzierte Prognose vernachlässigt zunächst einmal völlig die vielfältigen ethnischen, religiösen, kulturellen und politischen Unterschiede zwischen den verschiedenen Staaten in Ost- und Südostasien. Für die konkrete Markterschließung haben diese Unterschiede eine wesentliche Bedeutung und sollten vorab gut recherchiert werden. Im Rahmen dieses Beitrages wollen die Autoren angesichts der diskutierten Entwicklung jedoch drei Trends grob beschreiben, die nach unserer Auffassung in den nächsten Jahren die Entwicklung im asiatisch-pazifischen Raum bestimmen werden. Deutsche Unternehmen, die hierzu Lösungen anbieten können, haben unserer Meinung nach weiterhin gute Marktchancen in vielen Ländern Asiens.

1. Die Grundbedürfnisse - Essen, Wohnen, Arbeit - einer weiter wachsenden Bevölkerung in vielen Ländern Asiens müssen gesichert werden. Hierzu werden Lösungen benötigt in

- Bauwesen zur Errichtung vor allem der notwendigen Infrastruktur

- Rohstoffgewinnung, Energieerzeugung und -verteilung

- Produktion und Verarbeitung von Lebensmitteln

2. Die Erhöhung der Produktivität im exportorientierten, verarbeitenden Gewerbe wird auch die Sortimente der Unternehmen in asiatischen „Billiglohnländern“ verändern in Richtung höherwertiger Halbfabrikate, Komponenten, Fertigerzeugnisse und Dienstleistungen. Hierfür benötigen die asiatischen Unternehmen Lösungen in der Logistik, der Fertigung, der Meßtechnik, der Qualitätskontrolle u. a. mehr.

3. In den Mega-Millionen-Städten Asiens werden Lösungen u. a. in der Verkehrstechnik und im Umweltschutz benötigt, um die Lebensqualität in den Ballungszentren sowie die Basis des Wirtschaftswachstums ïberhaupt zu sichern.

Abschließend wollen die Frage diskutieren, ob sich aus den skizzierten Trends konkrete Marktchancen für brandenburgische Unternehmen entwickeln lassen. Dabei gilt es für die einzelnen Länder Asiens zu evaluieren, welche Priorität die Fragen der Infrastruktur, des Verkehrs und des Umweltschutzes in der Regierungspolitik haben werden, welche Vorhaben letztlich realisiert und finanziert werden sollen. Unternehmen, die hierzu Lösungen anbieten können, benötigen exzellente Kontakte zur jeweiligen Regierung, um

a) von Anfang an über die Ausschreibung dieser Vorhaben informiert zu werden und

b) bei der Realisierung von Aufträgen ggf. Importbeschränkungen für wichtige Komponenten aus Deutschland rasch überwinden zu können.
Angebote von Brandenburger Unternehmen in der Lebensmitteltechnik, der Logistik oder der Meßtechnik werden sich in den meisten Ländern Asiens, auch in der VR China, der Mongolei oder der VR Vietnam an private oder halbstaatliche Unternehmen richten. Nach unseren Erfahrungen sollte von Beginn der Geschäftskontakte an geklärt werden, ob derartige potentielle Partner in Asien verfügen über

a) Lizenzen zum Import ausländischer Dienstleistungen und Güter, sowie über

b) ausreichende finanzielle Bonität in Form von Devisenkrediten oder des Akzeptes von Akkreditiven, fuir welchen beispielsweise eine chinesische Bank bürgt.

Diese Fragen können z. T. schon durch qualifizierte Recherchen vor Beginn der Erschließung eines asiatischen Marktes geklärt werden. Wir erwähnten bereits, daß die einzelnen Länder Asiens im pazifischen Raum ganz unterschiedliche Kulturen haben. Die Differenzen zwischen den einzelnen Kulturen Asiens sind mindestens so groß wie zwischen den einzelnen Kulturen Europas. Für den Einstieg in einen asiatischen Markt halten wir aktuelle und detaillierte Informationen über Kultur, Politik und Wirtschaft des jeweiligen Landes für unbedingt notwendig. Derartige Vorabinformationen sind bereits in Deutschland in vielfältiger Weise nutzbar, wie der Erfahrungsbericht eines brandenburgischen Unternehmens der Lebensmitteltechnik über die Vorabrecherchen zur Erschließung des japanischen Marktes zeigt (vgl. THOMSEN 1997). Ein anderes brandenburgisches Unternehmen der Meßtechnik hatte in ähnlicher Weise 1992 mit der erfolgreichen Erschließung des Marktes in Indien begonnen. Diese beiden Unternehmen waren es auch, welche zusammen mit dem Institut für Internationales Marketing und Unternehmensfuihrung an der Technischen Fachhochschule Wildau im Jahr 1997 begonnen haben, den Markt in der VR China für sich zu erschließen (vgl. ausfuihrlich dazu der Beitrag von SCHWAB 1998 in diesem Heft).

\subsection{Der Weg nach Asien in kleinen, raschen Schritten}

Anläßlich des Wildauer Workshops am 8. Oktober 1997 wurde ein chinesisches Sprichwort aus ungenannter Quelle wie folgt widergegeben: „Eine lange Reise beginnt immer mit dem ersten Schritt".

In diesem Sinne skizzieren wir - nach den Erfahrungen unseres Forschungsprojektes - den Weg für kleine und mittlere brandenburgische Unternehmen auf asiatische Märkte wie folgt: Am Anfang sollte ein Markt in Asien konsequent ausgewählt und die notwendigen Länderinformationen recherchiert werden, so daß der Markt dann konsequent erschlossen und weiter bis zum Erfolg bearbeitet werden kann. Gerade kleine Unternehmen benötigen hierfür die Unterstützung erfahrener Handelshäuser oder Consultingbüros mit erstklassigen Kontakten vor Ort, die beispielsweise im Rahmen des Vermarktungshilfeprogramms der Bundesregierung angeboten werden kann. Nachdem in einem asiatischen Land ausreichend Referenzkunden gewonnen wurden, kann von dort aus die Erschließung weiterer asiatischer Märkte begonnen werden. 


\section{Literatur}

BfAi Bundesstelle für Außenhandelsinformation (1997): Bauwirtschaft in Asien spürt Zugluft; in: bfai-Info Asien, Heft 22/97

BMWi Bundesministerium für Wirtschaft (1996): Investitionschancen und Erfahrungen kleiner und mittlerer Unternehmen im asiatisch-pazifischen Raum; Bonn BMWi Bundesministerium für Wirtschaft (1997): Wirtschaft in Zahlen 1996; Bonn

Hünerberg, Reinhard (1994): Internationales Marketing; Landsberg/Lech

Könecke, Hans/Weickert, Sven/Wonneberger, Bernd (1997): Asien - Wachstumsmärkte für Brandenburger Unternehmen? in: Wissenschaftliche Beiträge der TFH Wildau, Heft 1/1997

Kulicke, Marianne (1993): Chancen und Risiken junger Technologieunternehmen; Heidelberg

LADVSTAT Landesamt für Datenverarbeitung und Statistik Brandenburg (1997): Außenhandelsstatistik des Landes Brandenburg für das Jahr 1996; Potsdam

Meissner, Hans Günther (1987): Strategisches Internationales Marketing; Berlin u.a.

Meyer, M. (1987): Die Beurteilung von Länderrisiken der internationalen Unternehmung; Berlin

Munkelt, Irmtrud (1996): Global Business für Mittelbetriebe - Ringen um Weltniveau; in asw Jg. 39, Heft 4/ 96, S. $32-37$

Neebe, Reinhard (1991): Überseemärkte und Exportstrategien in der westdeutschen Wirtschaft 1945 bis 1966; Stuttgart

Nieschlag, Robert; Dichtl, Erwin; Hörschgen, Hans (1991): Marketing; 16. Auflage, Berlin

Porter, Michael E. (1995): Wettbewerbsstrategie (Competitive Strategy); 8. Auflage, Frankfurt a. M./New York Stahr, Gunter (1993): Internationales Marketing; Ludwigshafen (Rhein)

StBA Statistisches Bundesamt (1997a): Statistisches Jahrbuch 1997 der Bundesrepublik Deutschland; Wiesbaden

StBA Statistisches Bundesamt (1997b): Statistisches Jahrbuch 1997 für das Ausland; Wiesbaden

Thomsen, Kerstin (1997): Erfolgreiche Markterschliessung in Asien; Beitrag zum Wildauer Workshop „Internationale Wachstumsmärkte“ am 8. Oktober 1997

\section{Verfasser}

\section{Prof. Dr. Bernd Wonneberger}

Dipl.-Psych. Hans Könecke

Technische Fachhochschule Wildau

Institut für internationales Marketing und Unter-

nehmensführung

Bahnhofstraße

15745 Wildau

Tel. +49 (0) 3375 - 508325

Fax +49 (0) $3375-508388$ 